\title{
Rural Women and Health: Experience from Gonoshasthaya Kendra
}

\section{Laila Parveen Banu}

\section{Introduction}

Women constitute 50 per cent of the population of Bangladesh and 92 per cent of the country's total population is rural. It is clear therefore, from these two statistics alone that when I refer to the woman in Bangladesh, I am thinking of the condition and status of a village dweller.

Through a combination of culture, poverty and ignorance a woman, through all stages of her life, is not a free and independent person but rather a male dominated and often oppressed creature. Rarely does she have any decision-making autonomy. From earliest childhood to puberty she is totally dependent on and subject to her father. This dependence on and subjection to a male is transferred to her husband when at an early age (by Western standards) she takes on the duties and responsibilities of married life. Having no social security, insurance permiums, etc., to provide for herself she must then, in the later years of her life, rely on her sons.

The Bengali woman therefore is not looked upon as one who acts and affects the lives of those around her in a free and independent manner, but as one who is constantly subject to the decisions of the male she is dependent upon at the various stages of her existence.

Let me illustrate with an example from a recent village clinic I was conducting. A young girl (15 years old) was brought in-a case of incomplete abortion - with high fever of several days duration but for whom no treatment had been sought. Case history taking revealed that she was the second wife taken by a man who already had children by his first wife. This girl was therefore not wanted to produce children but to provide extra labour. When she became ill through an unsterile abortion attempt, he did not want to spend the money to have her properly medically treated and so sent a message to her family to take her back to their house. Thus it was her own family who carried her to the clinic and then accompanied her during her stay in our hospital. However, when the husband heard that she had recovered he came to claim her back. If she had died, he would simply have taken another wife. A tragic, but unfortunately true and non-isolated instance of the lack of regard in which women are held. '

Woman, then, is accustomed to days of endless toil which leave her with still inadequate means of providing food and clothing for her husband and children. She sees her children sick with diarrhea; the rounded tummy, not indicative of robust health, but worms; the skeletal frame surrounding the bloated belly of malnutrition. Most likely she has seen one or more of her children die from these conditions, but what is she to do about it? The answer is not simple because it involves not only health care, but also socio-economic and educational considerations.

In all of this it is the woman who bears the burden of caring for her family, and so if anything is to be achieved by health care in Bangladesh, the problem must be met and solved where it originateswith the woman in the village. The Western medical education system (used almost universally in medical schools) tends to the rather myopic view of disease which indicates hospitalization and packaged medicine as the cure for the many ailments encountered in village health work. If this is the form of treatment used, what has been done to change the situation the patient will be returning to? Nothing. It is only by working to educate the people of the village themselvesmainly the women-in methods of sanitation, nutrition and management of common illnesses that the problem can be solved. The woman must be taught to identify the causes of her child's illness and to cope with them with whatever is available to her in the village.

\section{A health programme}

To achieve good village health care it would seem to me that three things are necessary: First, to alert the population to their health problems; second, to train primary health care workers to deal specifically with the villagers and these problems; and third, to search out ways to help them improve their economic situation.

The first two points are closely related and in this respect women have a vital role to play, not only in raising the standard of health care in the country, but also the status of women themselves. A successful programme must involve the villager. For this reason the ideal people to use in primary 
health care are the dais (traditional midwives) and young women with a minimal educational background. These are people who are a real part of the village scene. They have not been removed by years of Western-style education and urban living but are daily immersed in the life and problems of the village and are therefore much better able to communicate than the professional outsider.

Gonoshasthaya Kendra (Peoples Health Centre), itself a rural development project, was set up in 1972 with the aim of developing primary health care for rural areas. From the beginning we understood the position of women in rural areas and realised that any development without involving them would be fruitless. For this reason 75 per cent of our workers are women. We incorporate three categories of health workers-doctors, paramedics, and dais, each with a distinctive role to play in carrying out our programme. Being a rural health project we put particular emphasis on preventive medicine and family planning, which comprises about 75 per cent of our work load. Curative medicine on the other hand, accounts for only about 25 per cent.

The role of the doctor as we see it is this: first, to train and supervise the work of the paramedic. In this we use a six month to one year basic training period. Formal classes of instruction are given but closely integrated with practical village experience under the direct supervision of a doctor or senior paramedic. Equally important are the informal discussions between the doctor and paramedic about the situations she has encountered during a day's work in the village. Secondly, a doctor is always available as a back-up for any cases needing to be referred by a paramedic.

The most vital aspect of our rural health care is preventive medicine and family planning. This service is carried out by our paramedics working in close colaboration with the village dais. It is important to understand that the paramedics (primary health worker) and dai are not merely assistants to, or substitutes for, a physician in countries where there are not enough doctors to provide care for the total population. These people are professionals in their own right and have been trained specifically to work at a village level with the people-not in an urban centre or hospital. Therefore their skills and field of activity are quite distinct from that of the doctor.

Apart from our main Centre, there are six subcentres which senior paramedics are in charge of and which provide the base for their work. Their programme is essentially one of house to house visiting, discussing with individuals and groups the basics of sanitation, hygiene and nutrition. They spread awareness of the benefits of using clean water and simple, hygenic disposal of excreta. They endeavour to raise the skill of the villagers to deal with their own minor health problems. This could take several forms-a practical demonstration to a group of women on how to wash thoroughly and then administer medicine to a scabie-infested child, or how to disinfect a wound. A serious problem they must be taught to deal with is infant diarrhea. The packaged re-hydration fluid used in urban health centres and hospitals is certainly not available in the village, so the women must be taught to make their own preparation with tube-well water, sugar and salt. It was in following up just such a demonstration that one of our paramedics became aware of the importance of using simple techniques in her instructions. Inquiring of a woman why she had not made the fluid for her sick child as she had been taught, the women replied that she had no 'spoon' to measure the salt and sugar as done in the demonstration class. The paramedic too, is responsible for the immunisation programme-smallpox, DPT, BCG and tetanus toxoid-and for keeping accurate records of these. She advises on antenatal care, the importance of breast feeding and infant nutrition.

Apart from the health aspects, the paramedic also encourages the women to become involved in the vocational training activities carried on at our Centre and sub-centres. This provides them not only with a chance to learn useful skills but also to earn a small amount of money.

Family planning has and will continue to be a focal point of our health care. It is in this area that the village dais, in conjunction with the paramedic, plays such an important role. The dai is an accepted figure in any village and with proper instruction can do much to lower not only the birth rate, but also the infant and neo-natal death rate. The paramedic shares with them emphasis on the importance of washing the hands before delivery and boiling the thread before tying the umbilical cord. They also work together to inform, motivate and supply the women with the various contraceptives available.

Unfortunately, we again come up against the problem of male dominance. The Bengali woman is most often not free to decide for herself whether and/or when she will use contraceptives. Though she is the one who bears the burden not only of repeated pregnancy but also of child-rearing, she is subject to the decision of her husband in this 
matter. His consideration is not the welfare of his wife, but rather the fact that infant mortality is very high, and that by the time a child reaches the age of 6-7 he or she can be hired out to rich landowners and also help in the family fields. Thus it is essential in his eyes to have many children so that enough will survive to help supplement the family income by a few taka per month. We had the case of one young women who decided to defy her husband and take the injection, telling him it was a vaccine. He eventually became suspicious and having forced the truth from her, severely beat her. In addition to pill and injection contraceptives, our paramedics have also been trained to carry out the simple surgical tubal ligation independently under the supervision of a doctor.

\section{Socio-economics and education}

As was noted earlier, good village health care cannot be isolated from socio-economic and educational considerations. Not surprisingly it was again the social position of women that moved Gonoshasthaya Kendra to search out other options for women's employment.

"The greatest single cause of the tragedy of Bangladesh is the place that has been allotted by history, society and life itself to the women of the nation. Chained by culture, ignorance, fear, poverty, she is, in the words of a paramedic, "not a wife or a woman, but a slave". Hard and continuous labour with always the last portion of food, she cannot afford to fall sick. A slave unable to work is discarded and this would be her fate as well. Divorced, not accepted by her parents for whom she would be only another mouth to feed, and a disgrace, being separated from her husband, neither can she find employment to support herself. Now she can choose. As a beggar she can go to town and there discard the last shred of human dignity she may have had, or she can take the more attractive way out of insecticide poisoning..." (Chowdhury, 1976).

To try and save women from just this fate we inaugurated the inclusion of women into our vocational training programme.

We have carpentry, metal and shoe-making workshops attached to our programme. Why should these jobs be the perogative of men only? After all, the work is no more strenous than what the women would ordinarily be doing in their homes. Through the project we have proven that given the opportunity they can perform on a par with their male counterparts. Women are regularly being trained in such skills as carpentry and woodfinishing; plumbing; metal works and shoe making at our Centre. They also form the backbone of our jute and handicrafts project. Our aim in involving them thus could be considered two-fold. They are proving to their husbands, relatives and most importantly themselves that they are capable of performing such skills. Secondly, the contribution they make towards the family's total income from the stipend received for their work gradually enables them to take part in decision-making.

Fifty per cent of Bangladesh's population is made up of landless, marginal farmers. They have an acute problem to provide food for their families as they cannot afford to lease enough land to produce all they would need, and as the price of food escalates they are unable to purchase it in the market. Thus our agricultural loan scheme was introduced to try and help these exploited people. Naturally our efforts met with no little opposition from the land-owners and money-lenders. They were in danger of losing a most profitable business and did their level best to turn the people against the idea. However, after almost two years of trial, the response of the landless sharecroppers has shown that we are indeed moving in the right direction to be of service to them.

What of the children of those involved in our vocational training and agricultural loans? For the landless, every paisa is needed for food and shelter with nothing left over to provide the necessary clothing, books, etc., for schooling. Besides, their children are often used to tend livestock, carry water and firewood, and take meals to those working in the fields. So Gonoshasthaya Kendra's school was begun, but with unusual qualification requirements - the children must be of landless parents with no other educational opportunity. Time and consideration are given for the duties they are expected to perform at home. At school, they tend a small garden which, when studies are finished for the day, helps to provide their mid-day meal. The paramedic is also on hand here for instruction in basic health, hygiene and nutrition.

\section{Conclusion}

We are encouraged that those women directly or indirectly involved in our project feel proud and independent and experience their value as a member of the family. The birth rate among these women has already declined.

At the national level, women's involvement in our total programme has already made an impact. 
Field workers recently recruited to the Ministry of Population Control were all female, and the same is true of primary school teachers.

And so we continue in our small way to contribute to the revolt of women against their age-old oppression. Our programme has succeded in integrating women into all spheres of its activity and they are accepted and respected for what they have to contribute. Possibly an apt analogy would be that of a small stone dropped into the middle of a large lake-it will take a long time for the ripples to reach the edge, but at least the stone has been dropped.

\section{References}

Chowdhury, Zafrullah, 'The, Mother and Child in Bangladesh, a View from the People's Health Centre', Assignment Children, UNICEF, Geneva, January/March 1976 